\title{
$\bullet$ Cleft Lip/Cleft Palate Infants and Breastfeeding: A Survey
}

\section{IJCRR}

Section: Healthcare

ISI Impact Factor

(2019-20): 1.628

IC Value (2019): 90.81

$\operatorname{SJIF}(2020)=7.893$

(c) (i) (8)

Copyright@IJCRR

\section{Chattopadhyay D', Deol R*2, Rashim M³, Vathulya M}

'Associate Professor, Department of Burns \& Plastic Surgery, AllMS, Rishikesh., India; ${ }^{2}$ Assistant Professor, College of Nursing, AllMS,

Rishikesh, India; ${ }^{3}$ Senior Resident, Department of Burns \& Plastic Surgery, AllMS, Rishikesh, India; ${ }^{4}$ Associate Professor, Department of Burns \& Plastic Surgery, AllMS Rishikesh, India.

\section{ABSTRACT}

Introduction: Cleft lip/palate causes various issues, feeding being the major concern for parents. WHO recommends breastfeeding for 6 months. The cleft population is traditionally thought to be unable to breastfeed due to poor suckling. To deal with issues like inadequate gain in weight of infant due to feeding issues, trying out different feeding strategies and supplementation of calories through formula feeding, infants and mother often require assistance and guidance. Though the importance of human milk is well known, still breastfeeding is often discouraged for infants with cleft lip and palate. So, the researchers felt a need to explore breastfeeding practices among mothers of infants with cleft lip and /or palate.

Materials and Methods: A retrospective telephone survey design was used to collect information from mothers of children with cleft operated at tertiary care hospital, Uttarakhand. All the mothers of children with cleft who visited tertiary care centre, Uttarakhand over the past 3 years (April 2017 to March 2020) were included in the study. The total Enumerative technique was used and 122 mothers were interviewed. A structured questionnaire related to breastfeeding of the infant was used.

Results and Conclusion: The study yielded quite significant insights into breastfeeding practices in infants with a cleft. Only 15 infants received exclusively expressed breastmilk for 6 months. Only $21 \%$ of mothers received encouragement for breastfeeding. These findings can be utilised to support, counsel and encourage breastfeeding children with cleft.

Key Words: Breastfeeding, Infants, Cleft lip, Cleft palate

\section{INTRODUCTION}

Cleft lip and palate are congenital malformations affecting the lip, palate or both caused by an error in the facial fusion process during the embryonic period due to changes in the normal development of primary and/or secondary palate. Cleft lip/palate causes various issues, feeding being the major concern for parents. ${ }^{1}$ The rate of incidence of this anomaly varies from 0.28 to 3.74 per 1000 live births. ${ }^{2}$

WHO recommends breastfeeding for 6 months. The cleft population is traditionally thought to be unable to breastfeed due to poor suckling. To deal with issues like inadequate gain in weight of infant due to feeding issues, trying out different feeding strategies and supplementation of calories through formula feeding, infant and mother often require assistance and guidance. Feeding issues due to an infant's condition often result in maternal stress, increased anxiety among mothers and has a negative impact on mother-infant bond- ing. Breast milk provision is not given much importance as an outcome of the above-mentioned issues. Though the importance of human milk is well known, still breastfeeding is often discouraged for infants with cleft lip and palate. Inability to feed effectively can lead to maternal stress and anxiety as well as poor mother-infant bonding.,

So, the researchers felt a need to explore breastfeeding practices among mothers of infants with cleft lip and /or palate. A survey was conducted from a tertiary care centre to assess breastfeeding practices amongst mothers of cleft lip and palate. ${ }^{6,7}$

\section{MATERIALS AND METHODS}

Study Design: A retrospective telephone survey design was used to collect information from mothers of children with cleft operated at tertiary care hospital, Uttarakhand.

\section{Corresponding Author:}

Rupinder Deol, Assistant Professor, College of Nursing, AIIMS, Rishikesh, India.

Phone: 7055074477; Email: deolrains@gmail.co

ISSN: 2231-2196 (Print)

ISSN: 0975-5241 (Online)

Received: 21.11 .2020

Revised: 13.01 .2021

Accepted: 18.03 .2021

Published: 05.07 .2021 
Study Population and Duration: Only mothers of children with cleft were included as the study sample. All the mothers of children with cleft who visited tertiary care centre, Uttarakhand over the past 3 years (April 2017 to March 2020) were included in the study.

Exclusion Criteria: Mothers not willing to give informed consent for participation or not understanding either Hindi or English were excluded from the study.

Sampling and Sample size: Total Enumerative technique was used to collect information from mothers regarding breastfeeding practices in children with cleft. A total of 167 children were operated in the institute during the past three years. Out of these, a total of 122 mothers responded/gave consent for participation in the study and was interviewed. Mothers were explained about the maintenance of confidentiality.

Data collection tools: Primary sources of data included telephonically asking for socio-demographic information of mother and child and structured question related to breastfeeding of the infant with cleft lip/ palate or both. Structured tools were used for data collection.

Reliability and validity: Tools were validated and the reliability was checked. Tools were translated into the Hindi language, back-translated, and retranslated, followed by a pilot study on 5 mothers.

Ethical considerations: The study was initiated after due permission from the institutional ethics committee. Anonymity and confidentiality of data were maintained. Informed consent was taken from participants.

\section{Analysis \& Results}

The recorded data were analysed using appropriate statistical measures. Table 1 depicts the socio-demographic profile of mothers and their children with cleft. Details of the diagnosis of children are shown in Table 2. Important breastfeedingrelated information is depicted in Table 3.

Table 1: Socio-demographic profile of mothers/ children with cleft. $(\mathrm{N}=122)$

\begin{tabular}{llc} 
S. No. & Variable & Frequency (\%) \\
1. & Gender of Child & \\
& Male & $53(43)$ \\
& Female & $69(57)$ \\
2. & Education of Mother & \\
& Illiterate & $52(43)$ \\
& Literate & $39(32)$ \\
& Literate Plus & $31(25)$ \\
3. & Place of Delivery & \\
& At medical facility & $99(81)$ \\
& At home & $23(19)$ \\
\hline
\end{tabular}

Table 2: Percentage distribution of children as per their diagnosis. $(\mathrm{N}=122)$

\begin{tabular}{lc} 
Diagnosis & Frequency (\%) \\
Cleft Lip only & $36(30)$ \\
Cleft Palate only & $27(22)$ \\
Cleft Lip and Palate & $59(48)$ \\
\hline
\end{tabular}

Table 3: Breastfeeding related information. $\quad(\mathrm{N}=122)$

S. No. Variable Frequency (\%)

1. Received Breastfeeding advice within 7 days of birth

Yes

$105(86)$

No

$17(14)$

2. Breastfeeding advice received from: $(\mathrm{n}=105)$

Doctor/Nurse at the medical facility

$73(70)$

ASHA worker

$32(30)$

3. Gave colostrum to the baby

Yes

$52(43)$

No

$70(57)$

4. Type of feeding initiated for infant

Direct Breastfeed

29

Expressed breast milk

Formula feed

26

Further, none of the mothers continued direct breastfeeding for more than 15 days. Few of them switched over to expressed breast milk feeding. Out of the total, 82 mothers provided exclusively expressed breastmilk for 1 month. Only 15 infants received exclusively expressed breastmilk for 6 months. On average, infants received breastmilk for 2.28 months

Supplementation was done by formula feed/ cow's milk. Poor supply was cited as the most frequent challenge to providing breast milk and caused a cessation in $71.24 \%$ of mothers. A total of 36 infants was advised formula feeds due to poor weight gain.

As per the use of additional devices, none of the mothers used any additional special devices for feeding. Total 11 mothers, adopted modified football position for feeding their infants. Difficulties reported in feeding by mothers included poor supply, stress, difficulty in suckling, the increased time required. 


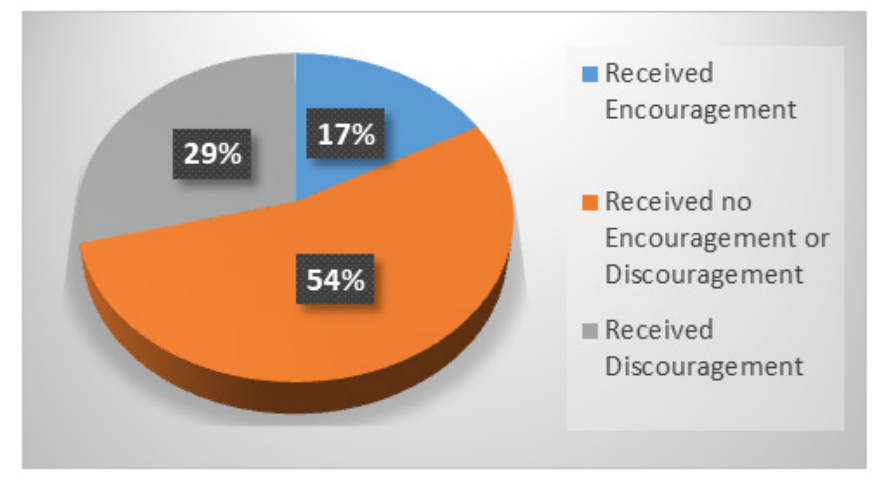

Figure 1: Family support received for breastfeeding by mothers.

As per family support, only 21 mothers (17\%) received encouragement, 66 mothers $(54 \%)$ received neither support nor discouragement while 35 mothers (29\%) were discouraged from breastfeeding by their family members. (Fig 1)

\section{DISCUSSION}

A pilot survey done by Kaye et al. on breastfeeding practices among children with Cleft Lip/Palate or both reported that $78 \%$ of mothers initiated either direct breastfeeding or expressed breast milk for their infants. The mean duration of human milk provision was 4 months. Most mothers (72\%) supplemented human milk feeding with formula either because of poor milk supply or was recommended for improved weight gain. The above findings are in lieu with current study findings, but the mother's milk was provided for a much shorter duration may be due to differences in support system. ${ }^{4,5}$

\section{CONCLUSIONS AND RECOMMENDATIONS}

The study yielded quite significant insights into breastfeeding practices in infants with a cleft. These findings can be utilised to support, counsel and encourage breastfeeding children with cleft. Support from a health care professional, particularly lactation specialist and nursing staff is critical. Breastfeeding and the use of human milk should be emphasized and prioritised. ${ }^{5}$ Further studies can be done on a larger sample size to generalise the findings.

\section{Financial Support and Sponsorship: Nil}

Conflicts of Interest: There are no conflicts of interest.

\section{REFERENCES}

1. Duarte GA, Ramos RB, Cardoso MC. Feeding methods for children with cleft lip and/or palate: A systematic review. Braz J Otorhin. 2016; 82(5):602-609.

2. Kumar MS, Vankayala B, Kumar M, Gudugunta L, Basavarajaiah JM et al. Evaluation of Feeding Practice in Infants with Cleft Lip and Palate at Cleft Centres. Den Sci. 2019;18 (7):14201427.

3. Trenouth MJ, Campbell AN. Questionnaire evaluation of feeding methods for cleft lip and palate neonates. Int J of Ped Dent. 1996; 6:241-244.

4. Kaye A, Cattaneo C, Huff M H, Staggs SV. A pilot study on mother's breastfeeding experiences in infants with cleft lip and/ or palate. Adv Neo care. 2019; 19 (2):127-137.

5. Burca NDL, Gephart MS, Miller C, Cote C. Promoting breast milk nutrition in infants with cleft lip and/or palate. Adv Neo Care. 2016; 16 (5):337-344.

6. Kaur N, Deol R, Yadav A. Correlation of feeding practices and health profile of children. Nurs J Ind. 2014 MayJun;105(3):128-130. PMID: 25643566.

7. Shetty MS, Khan MB. Feeding considerations in infants born with cleft lip and palate. APOS Trends Orthod 2016;6:49-53. 